\title{
A two-zone emission model for Blazars and the role of Accretion Disk MHD winds
}

\author{
Stella Boula, ${ }^{a, *}$ Apostolos Mastichiadis $^{a}$ and Demosthenes Kazanas ${ }^{b}$ \\ ${ }^{a}$ Department of Physics, National and Kapodistrian University of Athens, Panepistimiopolis, GR 15783 \\ Zografos, Greece \\ ${ }^{b}$ NASA Goddard Space Flight Center, Greenbelt, MD, United States \\ E-mail: stboula@phys.uoa.gr
}

Blazars are a sub-category of radio-loud active galactic nuclei with relativistic jets pointing towards the observer. They exhibit non-thermal variable emission, which practically extends over the whole electromagnetic spectrum. Despite the plethora of multi-wavelength observations, the origin of the emission in blazar jets remains an open question. In this work, we construct a twozone leptonic model: particles accelerate in a small region and lose energy through synchrotron radiation and inverse Compton Scattering. Consequently, the relativistic electrons escape to a larger area where the ambient photon field, which is related to Accretion Disk MHD Winds, could play a central role in the gamma-ray emission. This model explains the Blazar Sequence and the broader properties of blazars, as determined by Fermi observations, by varying only one parameter, the mass accretion rate onto the central black hole. Flat Spectrum Radio Quasars have a strong ambient photon fields and their gamma-ray emission is dominated by the more extensive zone, while in the case of BL Lac objects, the negligible ambient photons make the smaller, i.e. acceleration, zone dominant.

$37^{\text {th }}$ International Cosmic Ray Conference (ICRC 2021)

July 12 th - 23rd, 2021

Online - Berlin, Germany

\footnotetext{
${ }^{*}$ Presenter
} 


\section{Introduction}

Blazars (Flat Spectrum Radio Quasars and BL Lac objects) are the most extreme type of Active Galactic Nuclei (AGN), with relativistic jets pointing toward us. Their emission is non-thermal and it is amplified due to the relativistic bulk motion of the jets. Blazar Spectral Energy Distribution (SED) consists of two broad "humps", one spanning from radio to optical-UV (and occasionally $\mathrm{X}$-ray) bands and another one that extends from X-rays to multi-GeV and occasionally to $\mathrm{TeV} \gamma$ rays. The low-frequency component is believed to be due to synchrotron emission by non-thermal electrons, while the higher one is attributed to inverse Compton (IC) scattering of the relativistic electrons on synchrotron or external photons. Relativistic plasma motion and radiative cooling affect the temporal variability of these sources, which helps define the emitting region's size. Certain patterns in the spectral features became apparent and are now known as the "Blazar Sequence" [1]. Blazars become redder with increasing bolometric luminosity $L_{\mathrm{bol}}$, in that their synchrotron peak frequency $v_{\mathrm{pk}}^{\mathrm{syn}}$ decreases as $L_{\mathrm{bol}}$ increases; at the same time, their Compton dominance (CD; i.e., the ratio of their IC to synchrotron luminosities) increases and so do their $\gamma$-ray spectral indices, i.e. their spectral index becomes steeper. The Blazar Sequence, established originally with 132 objects out of which only 33 were detected in high energy $\gamma$-rays, was supplemented with the launch of Fermi and the discovery of more than $5000 \gamma$-ray Blazars as recorded in the 4th Fermi Blazar Catalog [2]. This compilation provided novel correlations that replaced those of the original Blazar Sequence. This result is implying that the underlying physics is probably related to fundamental parameters of the AGN phenomenology.

The optically thin Blazar $\mathrm{GeV}$ emission suggests its location to be far from the accreting black hole (BH), possibly out to a distance as large as $10^{6} R_{S} \sim 10 \mathrm{pc}$ [3] (where $R_{S}$ is the Schwarzschild radius ). Furthermore, the AGN torii (dusty, molecular structures of height/radius ratios $z / D \simeq 1$ ) invoked in the unification of the radio-quiet or radio-loud AGN subclasses [4], are of similar scales and, as we argue, play a significant role in Blazar physics. To reconcile the discrepancy of the torii geometry expected, [5] proposed that these torii are, in fact, MHD accretion disk winds [6, 7]; these are launched across the entire disk, from the $\mathrm{BH}$ vicinity of a few $R_{S}$ to the $\mathrm{BH}$ influence radius $D \sim(c / \sigma)^{2} R_{S} \sim 10^{6} R_{S} \sim 10 p c$ (for $M_{B H} \simeq 10^{8} M_{\odot}$ ). Furthermore, the discovery of Warm Absorbers (WA, blue-shifted absorption features) and their successful modeling as photoionized MHD winds that extend to $r \sim 10^{6} R_{S}[8,9]$, established the combined AGN WA-torii as a single entity. Finally, modeling the absorbers of the Galactic BH GRO 1655-40 with the same type of winds [10] suggests the possibility of their presence in any accreting $\mathrm{BH}$.

The physical properties of such MHD winds depend on a few parameters and their presence allowed us to reproduce a theoretical Blazar Sequence by varying only one parameter, namely the mass accretion rate, $[6,11]$. Here we will show the results of a two-zone emission model based on the extension of our previous works. However, now we assume that electrons are accelerated into a zone close to the central engine and escape and radiate into a larger region, which will refer as the cooling zone.

\section{Emission model-Scalings to mass accretion rate}

The broader morphology of the non-thermal Blazar SED depends on the ratio of the magnetic to photon energy densities. To calculate the former we assume some sort of equipartition with the 
accreting matter, the energy density. By assuming the power of the accretion $P_{\text {acc }}=\dot{m} \mathcal{M} L_{\text {Edd }}$, with $\dot{m}$ the mass accretion rate normalized to the Eddington one and $\mathcal{M}=M_{\mathrm{BH}} / M_{\odot}$, where $M_{\mathrm{BH}}$ is the mass of the black hole, one can calculate the magnetic field energy density at position z:

$$
U_{\mathrm{B}} \propto \eta_{\mathrm{b}} \dot{m} \mathcal{M}^{-1}
$$

where $\eta_{\mathrm{b}}$ is a proportionality constant. We assume for the external photon field that is related to photons which are scattered on accretion disk wind particles [6] and thus the external photon field density $U_{\text {ext }}$ in the jet frame has the form:

$$
U_{\mathrm{ext}}=\Gamma^{2} \mathrm{U}_{\mathrm{sc}} \propto \Gamma^{2} \epsilon \dot{m}^{\alpha+1} \mathcal{M}^{-1} \quad(\alpha=1 \text { for } \dot{m} \geq 0.1 \text { and } \alpha=2 \text { for } \dot{m}<0.1),
$$

where $\epsilon$ is the efficiency of the conversion of the accreting mass into radiation and $\Gamma$ is the bulk Lorentz factor of the source. We have assumed that the disk emits like a black body characterized by a temperature $T_{\text {disk }}$ in order to estimate the spectrum of the scattered photons. As we pointed in [6], all input parameters required for the calculation of the spectrum are scaled with $\dot{m}$ and $\mathcal{M}$. Using the above definitions for the physical properties of the source, we obtain the Blazar SED, by solving the coupled integro-differential kinetic equations of electrons and photons as described in [12]. We emphasize that according to relations 1,2 the basic parameters of the system of equations depends basically only on the mass accretion rate, $\dot{m}$.

Therefore, we assume a blob of plasma of radius $R$ where particles are accelerated with a characteristic timescale $t_{a c c}$, (e.g. [13]). In the case of the first order Fermi acceleration we have

$$
t_{\operatorname{acc}_{F I}} \geq 6\left(\frac{c}{u_{s}}\right)^{2} \frac{\lambda}{c} \simeq 6 \frac{r_{g} c}{u_{s}^{2}}
$$

where

$$
r_{g}=\frac{\gamma m c^{2}}{e B} .
$$

In our study, we re-write the Equation 3 as $t_{a c c} \propto \gamma A_{a c c}(\dot{m})$ taking into account the dependence on the mass accretion rate. By solving the set of equations we find there is an almost linear dependence between $t_{a c c}$ and $\dot{m}$ in order to explain the high peaked synchrotron blazars, see Figure 1.

\section{Particles escape: A two-zone emission model}

As a second step, we study a two zone model: particles accelerate in a zone closer to the central engine, then they escape to a larger volume further away and cool due to synchrotron and inverse Compton losses. The kinetic equation of electrons in the first zone (or acceleration zone) has the form:

$$
\frac{\partial n_{e_{I}}(\gamma, t)}{\partial t}+\frac{n_{e_{I}}(\gamma, t)}{t_{e s c_{I}}(\dot{m}, \gamma)}+\frac{\partial}{\partial \gamma}\left[\frac{\gamma}{t_{a c c}(\dot{m}, \gamma)} n_{e_{I}}(\gamma, t)\right]=\mathcal{L}_{s y n}(\gamma, t)+\mathcal{L}_{I C S}(\gamma, t) .
$$

The kinetic equation of relativistic electrons in the second zone (or cooling zone) has the form:

$$
\frac{\partial n_{e_{I I}}(\gamma, t)}{\partial t}+\frac{n_{e_{I I}}(\gamma, t)}{t_{e s c_{I I}}}=Q_{i n j}+\mathcal{L}_{s y n}(\gamma, t)+\mathcal{L}_{I C S}(\gamma, t),
$$



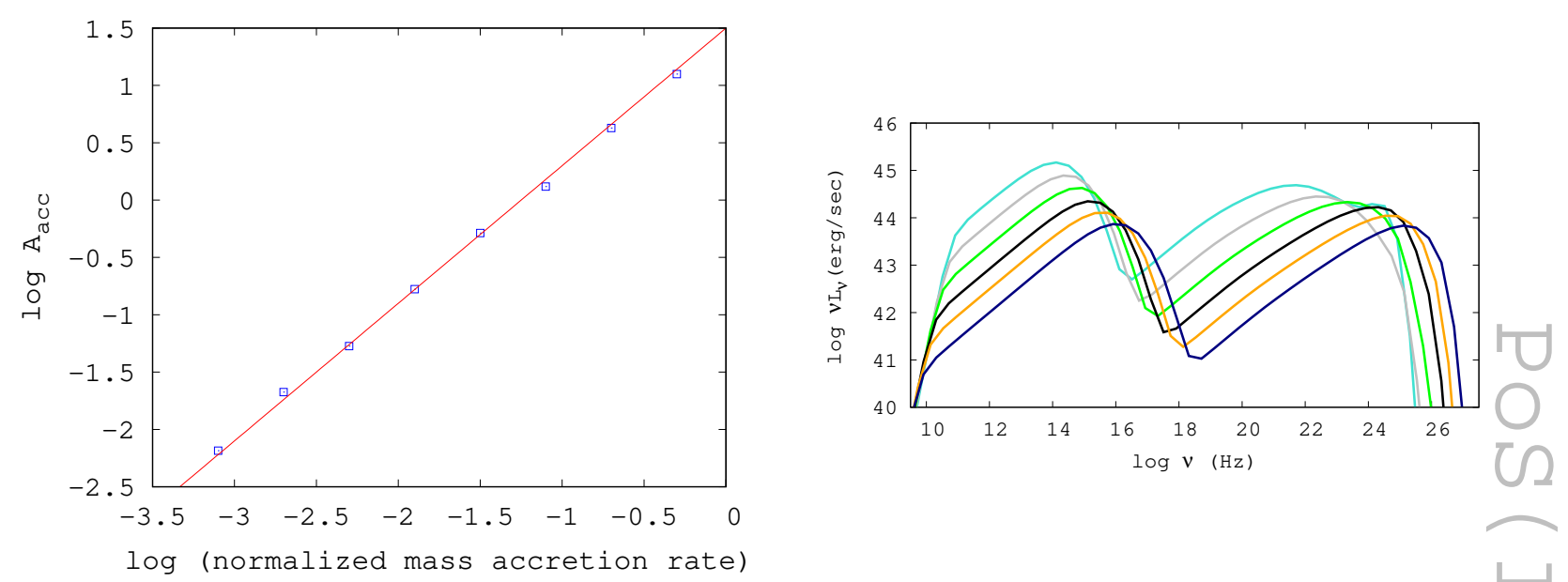

Figure 1: Left: The dependece of the acceleration timescale to the normalized mass accretion rate. Right: The calculated Spectral Energy Distribution of BL Lac objects in the case for various values of the mass accretion rate.

\section{Cooling zone}

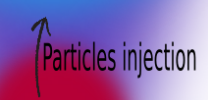

Acceleration zone

Figure 2: Sketch of the two-zone model. Particles accelerate and radiate in the acceleration zone (red). While, a fraction of the accelerated particles escapes into a larger volume(blue), where they cool and radiate. 


\begin{tabular}{|c|c|c|c|c|}
\hline$\dot{m}$ & $B(\mathrm{G})$ & $U_{\text {ext }}\left(\frac{\mathrm{erg}}{\mathrm{cm}^{3}}\right)$ & $A_{\text {acc }}$ & Blazar Class \\
\hline-0.5 & 1 & -2.6 & -4 & FSRQ \\
\hline-1.5 & 0 & -5.6 & -5 & LBL \\
\hline-2.5 & -1 & -8.6 & -6 & HBL \\
\hline
\end{tabular}

Table 1: The values of the input parameters for different mass accretion rates when particle acceleration is included in the numerical code. All the values are in a logarithmic scale.

where $n_{e_{I}}, n_{e_{I I}}$ the electron differential distribution function in the first and second zone respectively, $t_{e s c_{I}}, t_{\text {esc }_{I I}}$ the electron escape timescale from the first and the second zone respectively, the term $Q_{i n j}=\frac{n_{e_{I}}}{t e s c_{I}}$ refers to the relativistic electrons of the acceleration zone that escape and inject to the cooling zone, (e.g. [14]). To calculate synchrotron losses, we assume that the magnetic field decreases with the distance $z$ from the central engine as $B \propto 1 / z$. Furthermore, the energy density of the external photon field $U_{\text {ext }}$ is constant with the distance z, [6]. According to our assumption, the two classes of blazars show a very distinct behavior:

- BL Lac objects emission is dominated by the first zone. This results from the fact that the energy density of the magnetic field dominates in both zones. The reason is that in the acceleration zone, we have $U_{B_{I}}>>U_{\text {ext }}$ as BL Lac objects have a weak external photon field. In the radiation zone ${ }^{1}$ even the fact that the magnetic energy density $U_{B}$ decreases with z (see above), we still have that $U_{B_{I I}}>>U_{\text {ext }}$ everywhere, where $U_{B_{I I}}$ the magnetic field strength in the second zone, see Figure 3. As a result, the spectrum is dominated by the emission of the acceleration zone.

- FSRQs synchrotrom emission is mainly produced by the first zone, while the second zone is dominated by the inverse Compton scattering. For the parameter set that we study in the acceleration zone, the magnetic energy density is again more significant than the energy density of the external photon field. However, the second zone is further from the central engine, where the magnetic field decreases, but the external photons' energy density remains constant [6]. Now we have $U_{\text {ext }}>>U_{B_{I I}}$ and the contribution of the external inverse Compton scattering is more significant than this of the first zone. As a result, in the total flux, the low-frequency component is related to the synchrotron radiation from the acceleration zone and the high one to the inverse Compton scattering losses from the cooling zone.

The above can be illustrated by Figure 3 which depicts our calculations for the two zones in the cases of FSRQs and BL Lac objects.

In Figure 4 we present our results for a theoretical Blazar Sequence in the case of the two-zone model, see Table 1 for the values of the input parameters and the dependence of $\dot{m}$. In both classes of blazars particles escape from the acceleration zone. However, FSRQs present a characteristic signature in $\gamma$-rays, because particles inject into the cooling zone and interact with a strong external photon field. On the contrary, in BL Lac objects, when particles escape, they do not interact with

\footnotetext{
${ }^{1}$ The size of the second zone is related to the electrons cooling timescale, in order the losses to be Compton dominated.
} 


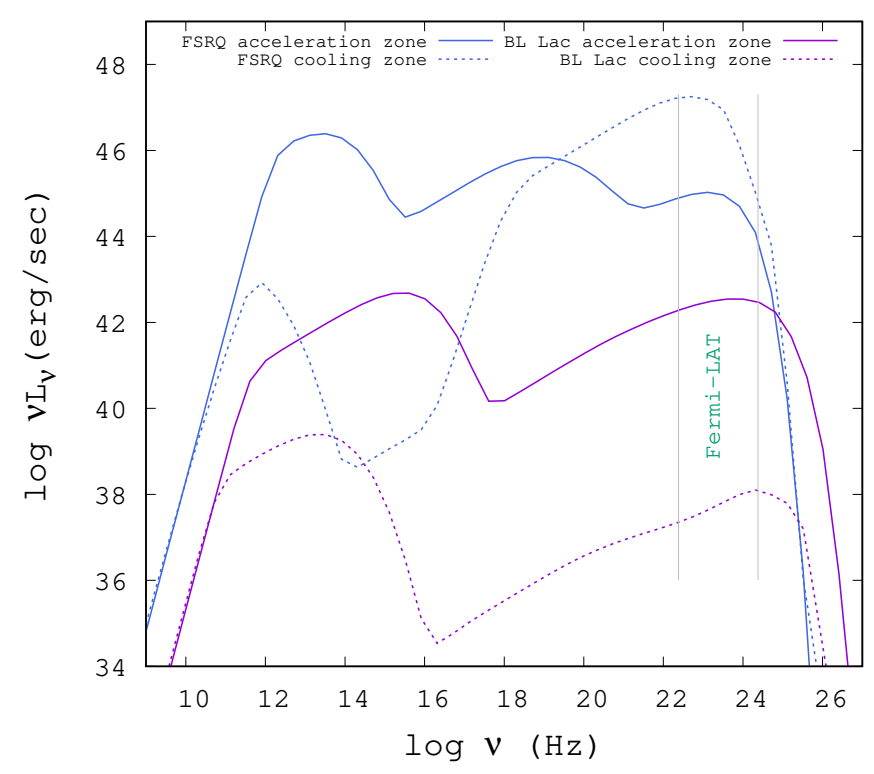

Figure 3: Results for FSRQ and BL Lac objects according to the two-zone model. Straight lines depict the emission from the acceleration zone and dotted lines the emission from the cooling zone.

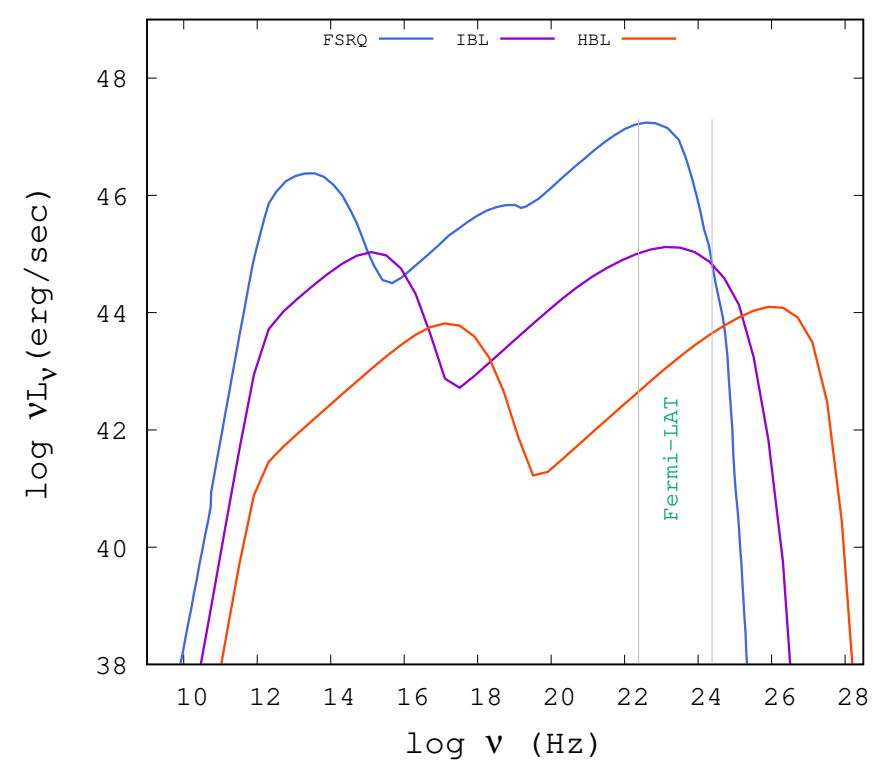

Figure 4: The theoretical Blazar Sequence according to the superposition of the two-zone emission by varying only the mass accretion rate, see Table 1 for the values of the input parameters. The acceleration zone is at a distance of $z=0.01 p c$ from the central black hole which is assumed to have a mass $\mathcal{M}=10^{8}$. The external photon field is produced from the isotropic scattering of disk photons on the wind particles between radii $R_{1}=9 \cdot 10^{14} \mathrm{~cm}$ and $R_{2}=3 \cdot 10^{18} \mathrm{~cm}$. The efficiencies of the magnetic field and the external photon field are $\eta_{\mathrm{b}}=0.01$, and $\epsilon=0.05$, respectively. The number of the injected electrons in the acceleration process, which is assumed to be of Type I Fermi, depends linearly on the mass accretion rate. The bulk Lorentz factor is $\Gamma=30$ and the Doppler factor is $\delta=15$. The characteristic temperature of the accretion disk is $T_{\text {disk }}=3 \cdot 10^{3} \mathrm{~K}$. 


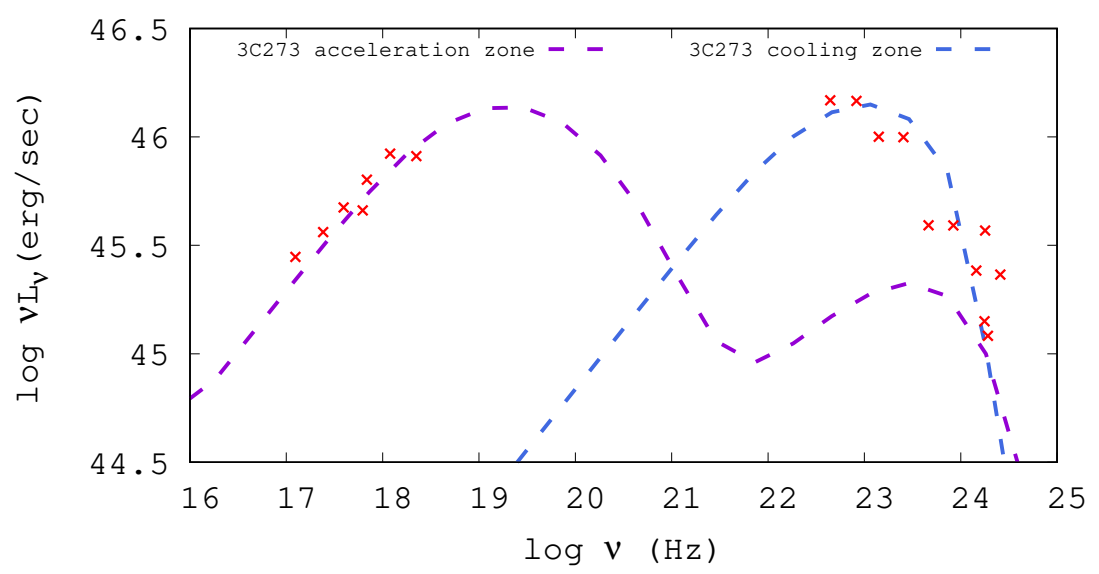

Figure 5: An application of the two-zone model in the case of FSRQ 3C273. The acceleration zone is at distance $z=0.01 p c$. The external photon field is produced from the isotropic scattering of disk photons on the wind particles between radii $R_{1}=9 \cdot 10^{14} \mathrm{~cm}$ and $R_{2}=3 \cdot 10^{18} \mathrm{~cm}$. The magnetic field strength in the acceleration zone is $B=1 G$, its radius is $R=5 \cdot 10^{15} \mathrm{~cm}$, and the energy density of the external photon field is $U_{\text {ext }}=2.5 \cdot 10^{-3} \frac{\mathrm{erg}}{\mathrm{sec}}$. The bulk Lorentz factor is $\Gamma=30$ and the Doppler factor is $\delta=15$. The characteristic temparature of the accretion disk is $T_{\text {disk }}=3 \cdot 10^{4} \mathrm{~K}$. Data are reproduced from [15].

a strong external photon field and as a result, the cooling zone has a lower contribution to the total flux.

In Figure 5 we show our results in applying a two-zone model in the case of 3C273, an FSRQ object. We zoom on the high-energy part of the spectrum (X-rays, $\gamma$-rays). X-rays are produced by SSC of the acceleration zone and $\gamma$-rays by the external Compton of the cooling zone.

\section{Conclusion}

In this work, we reproduce the theoretical Blazar Sequence based on the model of [6] by varying only the mass accretion rate that seems to explain the Blazar phenomenology. We solve self-consistently the electron and photon kinetic equations, by assuming that electrons accelerate into a small region and lose energy through synchrotron and inverse Compton scattering. While, a part escapes to a larger volume where they lose energy through the same physical processes. Under this assumption, we produce the theoretical Blazar Sequence by adding up the fluxes of the two zones in both the cases of FSRQs and BL Lac objects.

\section{References}

[1] G. Fossati, L. Maraschi, A. Celotti, A. Comastri and G. Ghisellini, A unifying view of the spectral energy distributions of blazars, 299 (1998) 433 [astro-ph/9804103]. 
[2] The Fermi-LAT collaboration, The Fourth Catalog of Active Galactic Nuclei Detected by the Fermi Large Area Telescope, arXiv e-prints (2019) arXiv:1905.10771 [1905.10771].

[3] A.P. Marscher, S.G. Jorstad, V.M. Larionov, M.F. Aller, H.D. Aller, A. Lähteenmäki et al., Probing the Inner Jet of the Quasar PKS 1510-089 with Multi-Waveband Monitoring During Strong Gamma-Ray Activity, 710 (2010) L126 [1001 . 2574].

[4] R.R.J. Antonucci and J.S. Miller, Spectropolarimetry and the nature of NGC 1068, 297 (1985) 621.

[5] A. Konigl and J.F. Kartje, Disk-driven hydromagnetic winds as a key ingredient of active galactic nuclei unification schemes, 434 (1994) 446.

[6] S. Boula, D. Kazanas and A. Mastichiadis, Accretion disc MHD winds and blazar classification, 482 (2019) L80 [1810 . 01796].

[7] J. Contopoulos and R.V.E. Lovelace, Magnetically Driven Jets and Winds: Exact Solutions, 429 (1994) 139.

[8] E. Behar, Density Profiles in Seyfert Outflows, 703 (2009) 1346 [0908. 0539].

[9] K. Fukumura, D. Kazanas, I. Contopoulos and E. Behar, Magnetohydrodynamic Accretion Disk Winds as X-ray Absorbers in Active Galactic Nuclei, 715 (2010) 636 [0910 . 3001].

[10] K. Fukumura, D. Kazanas, C. Shrader, E. Behar, F. Tombesi and I. Contopoulos, Magnetic origin of black hole winds across the mass scale, Nature Astronomy 1 (2017) 0062 [1702.02197].

[11] S. Boula, D. Kazanas and A. Mastichiadis, Mhd Accretion Disk Winds And The Blazar Sequence, in High Energy Phenomena in Relativistic Outflows VII, p. 9, July, 2019.

[12] A. Mastichiadis and J.G. Kirk, Self-consistent particle acceleration in active galactic nuclei., 295 (1995) 613.

[13] J.G. Kirk, F.M. Rieger and A. Mastichiadis, Particle acceleration and synchrotron emission in blazar jets, 333 (1998) 452 [astro-ph/9801265].

[14] J.G. Kirk, F.M. Rieger and A. Mastichiadis, Particle acceleration and synchrotron emission in blazar jets, 333 (1998) 452 [astro-ph/9801265].

[15] P. Giommi, G. Polenta, A. Lähteenmäki, D.J. Thompson, M. Capalbi, S. Cutini et al., Simultaneous Planck, Swift, and Fermi observations of X-ray and $\gamma$-ray selected blazars, 541 (2012) A160 [1108. 1114]. 